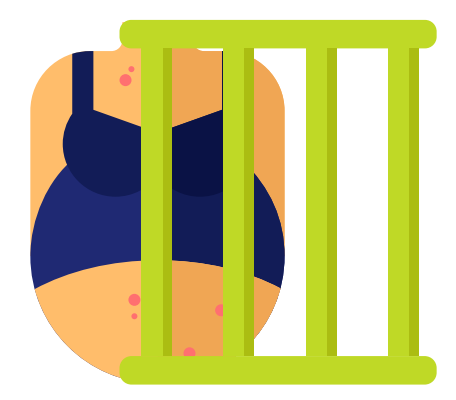

\title{
O DESAFIO DO COMBATE À SÍFILIS CONGÊNITA E À SÍFILIS EM GESTANTES NO SISTEMA PRISIONAL BRASILEIRO
}

\author{
THE CHALLENGE OF COMBATING CONGENITAL SYPHILIS AND SYPHILIS IN \\ PREGNANT WOMEN IN THE BRAZILIAN PRISON SYSTEM
}

\begin{abstract}
Eloiza da Silva Gomes de Oliveira
Professora Titular da Universidade do Estado do Rio de Janeiro (UERJ), possui Graduação em Psicologia e em Pedagogia, Especialização em Supervisão Educacional e Mestrado em Psicologia Escolar. Doutora em

Educação (Universidade Federal do Rio de Janeiro. E-mail: eloizagomes@hotmail.com

Janaína Luana Rodrigues da Silva Valentim

Possui Graduação em Secretariado Executivo (Faculdade de Ciências, Cultura e Extensão do RN), Especialização em Informática em Saúde (Universidade Federal do Rio Grande do Norte), Doutoranda em Estudos Contemporâneos - Centro de Estudos Interdisplinares do Século XX - Universidade de Coimbra.

E-mail: janaina.Irsv@gmail.com.

\section{Maíra Luciano Sidrim}

Possui Graduação em Engenharia de Produção (Faculdade Boa Viagem) e Mestrado em Engenharia de Produção (Universidade Federal do Rio Grande do Norte). E-mail: mairasidrim@gmail.com.

\section{Ronaldo Silva Melo}

Professor da Rede Estadual de Ensino do Estado do Rio de Janeiro (SEEDUC). Mestre em Psicologia Social e Professor convidado do Instituto Multidisciplinar de Formação Humana com Tecnologia da Universidade Estadual do Rio de Janeiro. E-mail: ronaldo@ifht.net.br

Sara Marisa da Graça Dias do Carmo Trindade

Professora no Departamento de História, Estudos Europeus, Arqueologia e Artes da Faculdade de Letras da Universidade de Coimbra. Doutora em História e pós-doutorado em Tecnologias Educacionais e da Comunicação (Universidade de Coimbra). E-mail:trindade.sara@gmail.com
\end{abstract}

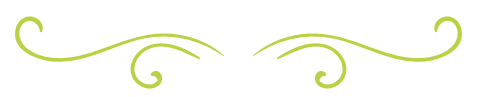

\section{RESUMO}

Diante do expressivo aumento nos casos de sífilis nos últimos anos no Brasil, este artigo tem como objetivo promover a reflexão sobre o combate à sífilis congênita e à sífilis gestacional no sistema prisional, por meio de uma revisão da literatura. A primeira etapa foi a busca de artigos científicos nas principais bases de dados, seguida da seleção dos artigos que abordavam o cenário da sífilis congênita e sífilis em gestantes no ambiente prisional e, por fim, análise das principais contribuições desses estudos para o tema em questão. Por meio da revisão da literatura, constatou-se que as mulheres privadas de liberdade são uma população 
chave para o combate à sífilis congênita e à sífilis em gestantes, considerando os elevados números de prevalência da doença nos estudos realizados com esse grupo, principalmente, quando comparados aos dados de mulheres livres. De acordo com os resultados deste artigo, para reduzir de forma consistente a prevalência de sífilis em gestantes, a taxa de transmissão vertical de sífilis e a incidência de sífilis congênita no ambiente prisional é preciso garantir que todas as gestantes privadas de liberdade tenham acesso a um pré-natal adequado, incluindo todas as ações de combate à sífilis oferecidas às gestantes livres.

Palavras-chave: Sífilis Congênita. Sífilis em Gestantes. Mulheres Privadas de Liberdade. Sistema Prisional Brasileiro.

\section{ABSTRACT}

Given the significant increase in syphilis cases in recent years in Brazil, this article aims to reflect on the actions against congenital syphilis and gestational syphilis in the prison system. For this, a literature review was carried out. The first step was searching for scientific articles in the main databases. Subsequently, articles that addressed the scenario of congenital syphilis and syphilis in expectant women in the prison environment were selected. Lastly, the fundamental contributions of these studies to the topic in question were analyzed. Through the literature review, it was observed that women deprived of their liberty represent a key population for combating congenital syphilis and syphilis in expectant women. It occurs because of the elevated number of disease prevalence in studies conducted within this group, especially when compared to data from free women. Therefore, to consistently reduce the prevalence of syphilis, its vertical transmission rate, and the incidence of congenital syphilis in the prison environment, it is necessary to ensure that all pregnant women deprived of liberty have access to adequate prenatal care, which includes all the actions to combat the disease as offered to free expectant women.

Key-words: Congenital Syphilis. Syphilis in Pregnant Women. Women Deprived of Liberty. Brazilian Prison System.

\section{INTRODUÇÃO}

Nos últimos anos o Brasil apresentou um aumento expressivo no número de casos de sífilis. De acordo com o Boletim Epidemiológico de Sífilis, no período entre 2010 e 2018 a taxa de incidência de sífilis congênita aumentou 3,8 vezes, passando de 2,4 para 9,0 casos por mil nascidos vivos e a taxa de detecção de sífilis em gestantes aumentou 6,1 vezes. Só no ano de 2018, foram notificados 26.219 casos de sífilis congênita e 62.599 casos de sífilis em gestantes (BRASIL, 2019).

A sífilis congênita é causada pela disseminação do Treponema pallidum, da gestante infectada para o seu concepto, por via transplacentária. Apesar de a transmissão vertical da sífilis ocorrer em qualquer fase gestacional ou estágio clínico da doença materna, os principais fatores que contribuem para a transmissão são o estágio da doença na mãe e a duração da exposição do feto no útero. (BRASIL, 2006).

De acordo com as diretrizes do Ministério da Saúde, a medida mais efetiva de controle da sífilis congênita consiste na garantia de uma assistência pré-natal adequada para todas as gestantes, o que inclui: captação precoce da gestante para o início do pré-natal; realização de pelo menos seis consultas com atenção integral qualificada; realização do VDRL no primeiro trimestre da gestação; instituição do tratamento adequado da gestante e de seu(s) parceiro(s); documentação dos resultados das sorologias e tratamento da sífilis na carteira da gestante; notificação dos casos de sífilis congênita. Além disso, as medidas de controle devem abranger outros momentos como a realização do parto e os procedimentos pós- aborto. (BRASIL, 2006). 
De um modo geral, a melhor estratégia para combater a sífilis congênita é o diagnóstico precoce da sífilis durante a gravidez juntamente com o tratamento rápido e adequado da gestante infectada. Nesse sentido, o estudo de Matthias et al. (2017) aponta que esse tipo de conduta evitou que muitas gestantes infectadas tivessem um bebê com sífilis congênita. Os atores afirmaram ainda que para erradicar a sífilis congênita provavelmente seria necessário a prevenção da sífilis entre todas as mulheres.

Diante desse contexto, as autoridades brasileiras vêm reunindo esforços para o enfrentamento da sífilis. Com o objetivo de promover ações de fortalecimento institucional para reduzir a incidência de sífilis no Brasil, em 2017 o Ministério da Saúde e a Universidade Federal do Rio Grande do Norte firmaram o Termo de Execução Descentralizada - TED - 54/2017, intitulado Projeto Resposta Rápida a Sífilis. O projeto realiza um conjunto de ações voltadas para o combate e controle da sífilis por meio do trabalho colaborativo entre a referida universidade e o Sistema Único de Saúde (SUS), atuando em sete áreas de cooperação: Vigilância, Gestão e Governança, Cuidado Integral, Educação, Comunicação, Pesquisa e Cooperações nacionais e internacionais.

Um dos grupos chave para o combate à sífilis no Brasil é a população privada de liberdade visto que, de acordo com os dados do INFOPEN, no período de 2017 foram notificados 1.095 casos de sífilis, gerando uma incidência de 4,72\% para esse grupo. Para o mesmo período foram registrados 196 casos de sífilis em mulheres privadas de liberdade, com incidência de $19,41 \%$. Para os homens privados de liberdade foram notificados 899 casos de sífilis, gerando uma taxa de incidência de 4,05\% (BRASIL, 2017).

Correa et al. (2017), corroboram o que foi dito acima: o sistema prisional brasileiro apresenta uma alta prevalência de sífilis, especialmente, entre mulheres presas. Os autores afirmam que é preciso estratégias de controle mais intensivas como triagem de IST, tratamento, educação em saúde e distribuição de preservativos para esse grupo.

Souza (2017) destaca que as ações de promoção à saúde também devem ser ofertadas para as pessoas privadas de liberdade, o que demanda ações estratégicas de saúde para os internos do sistema prisional, incluindo a articulação entre as esferas governamentais e entre a gestão da saúde e a justiça. Como sugestões o autor indica: a ampliação do diagnóstico da sífilis na admissão e rotina das unidades penais, atividades contínuas de educação em saúde para os internos e a capacitação dos profissionais de saúde que compõem a equipe da justiça.

Com o objetivo garantir o acesso ao cuidado integral no Sistema Único de Saúde para as pessoas privadas de liberdade, em 2014 foi criada a Política Nacional de Atenção Integral à Saúde das Pessoas Privadas de Liberdade no Sistema Prisional - PNAISP. Dentre as diretrizes da PNAISP destacam-se: atenção integral, contínua e de qualidade às necessidades de saúde da população privada de liberdade, controle e/ou redução dos agravos mais frequentes que acometem a população privada de liberdade e intersetorialidade para a garantia do direito à saúde para esse grupo (BRASIL, 2014). Constata-se, portanto, que o acesso aos serviços de saúde é um direito garantido para os brasileiros privados de liberdade. Entretanto, a alta incidência de sífilis, discutida anteriormente, indica que ainda existe um caminho a ser percorrido para que esse grupo tenha, de fato, acesso aos serviços de saúde.

No contexto das mulheres privadas de liberdade, a situação é ainda mais complexa visto que envolve outros fatores como gravidez, parto, amamentação e a maternidade de um modo geral. De acordo com as Diretrizes para a Convivência Mãe-Filho/a no Sistema Prisional, a todas as gestantes deve ser assegurado o acesso à primeira consulta de pré-natal o mais precoce possível, com a oferta de teste rápido para HIV e sífilis no primeiro e no terceiro trimestres, além 
dos demais exames pré-natais recomendados pelo Ministério da Saúde. Além disso, as gestantes deverão receber recomendações sobre dieta e devem ser garantidas a suplementação vitamínica e alimentação adequada, orientada por nutricionista, para cada etapa da gestação. (BRASIL, 2016).

Nesse sentido, um dado importante é que, segundo o INFOPEN Mulheres, apenas 14,2\% das unidades prisionais brasileiras que recebem mulheres possuem um espaço reservado para gestantes e lactantes (BRASIL, 2017), o que indica novamente um descompasso entre os direitos garantidos e a realidade oferecida às pessoas privadas de liberdade. Diante desse cenário, torna-se mais significativo o objetivo deste artigo, que é investigar o combate à sífilis congênita e à sífilis em gestantes no sistema prisional, por meio de uma revisão da literatura.

A vulnerabilidade feminina se expressa fortemente na situação de privação de liberdade. Entre 2012 e 2014 a FIOCRUZ realizou uma pesquisa intitulada "Nascer na Prisão", com uma população de população de 495 mulheres, 206 gestantes e 289 mães. Os resultados foram impactantes, no que se refere à atenção, à gestação e ao parto durante o encarceramento. Temos, por exemplo, que: 81\% quando já estavam grávidas; $55 \%$ das grávidas passaram por menos consultas de pré-natal do que o recomendado, 32\% não foram testadas para sífilis; mais de um terço das mulheres presas grávidas relataram o uso de algemas na internação para o parto. Maria do Carmo Leal e outros, que coordenaram o projeto, afirmam que: "O encarceramento amplia a vulnerabilidade social, individual e programática desta população, dificulta o acesso aos serviços de saúde seja para prevenção, assistência ou vigilância, bem como compromete o bem estar e o exercício pleno da cidadania" (LEAL et al., 2016, p. 2062).

\section{MÉTODOS}

De acordo com Ogburn e Nimkoff (apud LAKATOS; MARCONI, 1991, p. 20) a ciência é reconhecida por três critérios: a confiabilidade do seu corpo de conhecimentos, sua organização e seu método. $\bigcirc$ conhecimento científico é resultado da pesquisa ou investigação científica, através da aplicação de métodos e técnicas.

Segundo Gewandsznajder (1989, p. 12), "o que melhor caracteriza o conhecimento científico não é o que ele estuda, mas como estuda". Em sentido complementar, Gil (1999, p. 26) afirma que a investigação científica depende de um "conjunto de procedimentos intelectuais e técnicos" para que seus objetivos sejam atingidos: os métodos científicos. Assim, vamos falar das etapas metodológicas da investigação realizada.

A primeira etapa foi a busca de artigos científicos relacionados ao tema combate à sífilis congênita e à sífilis em gestantes no sistema prisional. Assim, realizou-se uma pesquisa nas bases de dados Pubmed, Scielo e Periódicos Capes. Em seguida, foram selecionados apenas os artigos científicos que de fato abordam o tema em questão.

O Quadro 1 apresenta os resultados das buscas realizadas em cada base de dados e a quantidade de artigos selecionados em cada busca. 


\begin{tabular}{|c|c|c|c|c|}
\hline Base de dados & $\begin{array}{c}\text { Combinação de palavras } \\
\text { pesquisadas }\end{array}$ & Filtros & $\begin{array}{l}\text { Quantidade de } \\
\text { estudos encontrados }\end{array}$ & $\begin{array}{l}\text { Quantidade de } \\
\text { estudos selecionados }\end{array}$ \\
\hline Pubmed & "congenital syphilis" AND "prisons"; & $x$ & 10 & 4 \\
\hline Pubmed & $\begin{array}{l}\text { "syphilis" AND "prisons" } \\
\text { AND "pregnant" }\end{array}$ & $x$ & 11 & 3 \\
\hline Pubmed & $\begin{array}{c}\text { "syphilis" AND "prison system" } \\
\text { AND "pregnant" }\end{array}$ & $x$ & 4 & 1 \\
\hline Pubmed & $\begin{array}{l}\text { "congenital syphilis" AND } \\
\text { "prison system". }\end{array}$ & $x$ & 1 & 0 \\
\hline Scielo & "congenital syphilis" AND "prisons" & $x$ & 3 & 3 \\
\hline Scielo & $\begin{array}{l}\text { "syphilis" AND "prisons" } \\
\text { AND "pregnant" }\end{array}$ & $x$ & 4 & 3 \\
\hline Periódicos Capes & "congenital syphilis" AND "prisons & $x$ & 108 & 3 \\
\hline Periódicos Capes & "syphilis"AND "prisoners pregnant" & É (exato) & 29 & 0 \\
\hline
\end{tabular}

Quadro 1 - Resultados das buscas de artigos nas bases de dados

Fonte: autoria própria (2020).

Por meio do Quadro 1, constata-se a escassez de artigos que abordam o combate à sífilis congênita e à sífilis em gestantes no sistema prisional. Além da baixa quantidade de artigos em cada busca, o número de artigos selecionados é consideravelmente menor. Vale ressaltar que muitos dos artigos selecionados estavam presentes em várias buscas, por isso apenas 5 estudos foram selecionados para análise.

Por fim, realizou-se uma análise detaIhada sobre as principais contribuições de cada artigo para a literatura dos temas em questão. Esses resultados estão apresentados no tópico seguinte.

\section{RESULTADOS E DISCUSSÕES}

O Quadro 2 apresenta os autores e ano de publicação, títulos dos artigos, periódicos nos quais os estudos foram publicados e países onde as pesquisas foram realizadas. 


\begin{tabular}{|l|l|l|l|}
\hline \multicolumn{1}{|c|}{ Autores / ano } & \multicolumn{1}{|c|}{ Título } & \multicolumn{1}{c|}{ Periódico } \\
\hline Domingues et al. (2017) & $\begin{array}{l}\text { Prevalence of syphilis and HIV infection during } \\
\text { pregnancy in incarcerated women and the incidence } \\
\text { of congenital syphilis in births in prison in Brazil }\end{array}$ & $\begin{array}{l}\text { Cadernos de Saúde } \\
\text { Publica }\end{array}$ & Brasil \\
\hline Batista et al. (2020) & $\begin{array}{l}\text { Alta prevalência de sífilis em unidade prisional } \\
\text { feminina do Nordeste brasileiro }\end{array}$ & Einstein (São Paulo) & Brasil \\
\hline Blank et al. (1997) & $\begin{array}{l}\text { New approaches to syphilis control. Finding } \\
\text { opportunities for syphilis treatment and congenital } \\
\text { syphilis prevention in a women's correctional setting. }\end{array}$ & $\begin{array}{l}\text { Sexually Transmitted } \\
\text { Diseases }\end{array}$ & EUA \\
\hline $\begin{array}{l}\text { Gouvêa, Corrêa e } \\
\text { Santos (2019) }\end{array}$ & $\begin{array}{l}\text { Incidência de sífilis e HIV em gestantes privadas de } \\
\text { liberdade no Rio de Janeiro }\end{array}$ & $\begin{array}{l}\text { Brazilian Journal of } \\
\text { Health Review }\end{array}$ & Brasil \\
\hline Gétaz et al. (2017) & Congenital syphilis in 2 children in a Bolivian prison. & $\begin{array}{l}\text { Revista Española de } \\
\text { Sanidad Penitenciaria }\end{array}$ & Bolívia \\
\hline
\end{tabular}

Quadro 2 - Artigos selecionados

Fonte: autoria própria (2020).

Por meio do Quadro 2, constata-se que 3 das pesquisas selecionadas para análise foram realizadas no Brasil e 2 foram realizadas em outros países da América. Outro ponto importante é que o primeiro desses estudos foi realizado em 1997 nos EUA e só em 2017 surge uma publicação brasileira acerca do tema. Por outro lado, após o primeiro estudo, o Brasil publicou uma sequência de mais 2 artigos em 3 anos.

$\mathrm{O}$ estudo de Domingues et al. (2017) avaliou, dentre outros aspectos, a prevalência de infecção de sífilis na gravidez, a transmissão vertical de sífilis e a incidência de sífilis congênita em filhos de mulheres encarceradas no Brasil. Segundo os autores, a prevalência estimada de sífilis durante a gravidez, para mulheres encarceradas, foi de 8,7\%, enquanto a estimativa de transmissão vertical nas mesmas foi de $66,7 \%$, com incidência de sífilis congênita de 58,1 por 1.000 recém-nascidos vivos.

O estudo apontou diferenças significativas ao comparar esses resultados com o cenário de mulheres não encarceradas que apresentaram os seguintes dados: prevalência de sífilis durante a gravidez em 1,3\% das mulheres, taxa de transmissão vertical de sífilis de $36,8 \%$, com uma incidência de sífilis congênita de 4,6 para 1.000 recém-nascidos vivos. (DOMINGUES et al., 2017).

Visando determinar a prevalência de sífilis e os fatores de risco associados ao sistema prisional feminino, Batista et al. (2020) realizou um estudo transversal com 113 mulheres de um Centro de Reeducação Feminino localizado na Região Nordeste do Brasil. Por meio de testes rápidos de sífilis, os autores estimaram a prevalência de sífilis em 22,1\% desse grupo de mulheres, enquanto a prevalência de sífilis em gestantes foi de 28,6\%. Por meio de um formulário, para avaliar situações de risco para infecções sexualmente transmissíveis, foi verificada relação estatisticamente significativa entre a infecção pela sífilis e a história prévia de infecções sexualmente transmissíveis, mas a maioria das participantes diagnosticadas com a doença desconhecia um histórico de infecção sexualmente transmissível nos últimos 12 meses.

Segundo os autores, a prevalência de sífilis na população carcerária feminina estudada foi alta, principalmente entre as gestantes, o que reforça a necessidade de estabelecer medidas preventivas e de tratamento da doença, bem como cuidados pré-natais adequados no ambiente prisional. $\bigcirc$ estudo sugere ainda medidas como diagnóstico precoce no período de admissão das mulheres na unidade prisional e atividades de conscientização em termos de melhoria da qualidade de vida da população encarcerada para minimizar o impacto dasífilis nos sistemas prisionais e, consequentemente, melhorar esse indicador de saúde no país (BATISTA et al., 2020).

A pesquisa de Blank et al. (1997) constatou que 17 (31\%) das 55 mulheres grávidas do 
sistema prisional de Nova York tinham indicação para o tratamento de sífilis e o Sistema de Vigilância de Sífilis Congênita identificou seis (35\%) pares mãe-bebê por sorologias reativas à sífilis no momento do parto. Segundo os autores supracitados, a triagem local e o uso de registros de casos de sífilis, aliados aos testes laboratoriais tradicionais, podem melhorar a eficácia do tratamento da sífilis nas populações de presos. A alta taxa de infecção detectada indica a necessidade de expandir os esforços para a rapidez na detecção e tratamento de sífilis nas instalações penitenciárias dessas mulheres.

Por meio de um estudo de coorte, pesquisa observacional de caráter epidemiológico que objetiva observar, em uma população definida, a incidência de determinada doença ou fenômeno associado à saúde ou à doença, Gouvêa, Corrêa e Santos (2019) avaliaram a frequência da soropositividade para sífilis e para o HIV em gestantes de uma unidade prisional do Rio de Janeiro. As 18 gestantes que participaram dessa pesquisa haviam realizado o teste para rastreamento de Sífilis e HIV, mas apenas $50 \%$ possuíam resultado do exame para sífilis e 55,55\% do exame para HIV. Das que apresentavam resultado para sífilis, 22,22\% apresentaram resultado positivo. Segundo os autores, o protocolo para o rastreamento da Sífilis e do HIV foi cumprido para todas as gestantes, mas a demora na disponibilidade do resultado interferiu no diagnóstico precoce e tratamento imediato.

Segundo as autoras, a taxa de incidência da Sífilis e do HIV em gestantes privadas de liberdade ainda é alta, o que demanda uma ampliação da assistência em termos de diagnóstico e tratamento, principalmente no pré-natal. Assim, para intervir de forma eficaz no controle da sífilis congênita e da transmissão vertical do vírus HIV no sistema prisional, se faz necessário fortalecer as ações de prevenção e tratamento precoce dessas infecções no sistema prisional brasileiro e um dos caminhos apontados pelas autoras é a intensificação da Política Nacional de Atenção Integral à Saúde das Pessoas Privadas de Liberdade no Sistema Prisional (GOUVÊA; CORRÊA; SANTOS, 2019).

Gétaz et al. (2017) realizaram um estudo transversal em 2013, identificando 28 casos de sífilis ativa entre 219 mulheres privadas de liberdade em uma prisão de Cochabamba, na Bolívia. Além disso, os autores realizaram uma busca por sífilis congênita entre as crianças que moravam com as mães infectadas e 2 dessas crianças foram diagnosticadas com a doença. De acordo com os autores anteriormente citados, os casos de sífilis congênita enfatizam a relevância da prevenção da transmissão vertical da sífilis por meio de triagem sistemática de gestantes e tratamento adequado por profissionais com treinamento específico para atender a população carcerária, visto que esse grupo representa uma população-chave para o controle de sífilis.

Quadro 3 a seguir apresenta um compilado dos principais aspectos investigados no que se refere à sífilis em gestantes e sífilis congênita nos artigos analisados.

\begin{tabular}{|c|c|c|c|}
\hline Autores / ano & $\begin{array}{l}\text { Abrangência } \\
\text { do estudo }\end{array}$ & $\begin{array}{c}\text { Percentual de sífilis em } \\
\text { gestantes privadas } \\
\text { de liberdade }\end{array}$ & $\begin{array}{c}\text { Sífilis congênita em filhos } \\
\text { de mulheres privadas } \\
\text { de liberdade }\end{array}$ \\
\hline Domingues et al. (2017) & Nacional & $8,7 \%$ & $66,7 \%$ \\
\hline Batista et al. (2020) & Regional & $28,6 \%$ & X \\
\hline Blank et al. (1997) & Municipal & $31 \%$ & $35 \%$ \\
\hline Gouvêa, Corrêa e Santos (2019) & Uma unidade prisional & $22,22 \%$ & $x$ \\
\hline Gétaz et al. (2017) & Uma unidade prisional & X & 02 crianças diagnosticadas \\
\hline
\end{tabular}

Quadro 3 - Aspectos abordados nos artigos analisados

Fonte: autoria própria (2020). 
Por meio do Quadro 3, observa-se uma variação nos estudos entre nacional, regional, municipal e local, o que dificulta a comparação entre os seus resultados. Quanto ao percentual de sífilis em gestantes privadas de liberdade, além da variação observada nos resultados. Dessa forma, se faz necessário destacar que esses dados são preocupantes visto que, mesmo considerando o percentual mais baixo encontrado, que foi de $8,7 \%$, esse número é bem mais alto que a prevalência de sífilis em mulheres grávidas não encarceradas que, de acordo com o estudo de Domingues et al. (2017), foi de 1,3\%. Vale ressaltar que o estudo de Gétaz et al. (2017) não disponibilizou número e percentual de mulheres grávidas privadas de liberdade, por isso esse dado não consta no Quadro1.

Com relação às informações sobre sífilis congênita, observa-se uma variedade ainda maior visto que os estudos analisados utilizaram indicadores diferentes. Domingues et al. (2017) detectaram uma taxa de 66,7\% de transmissão vertical entre as gestantes diagnosticadas com sífilis e incidência de sífilis congênita de 58,1 por 1.000 recém-nascidos vivos. Blank et al. (1997) constataram uma taxa de transmissão vertical de 35\%. O estudo de Gétaz et al. (2017) não disponibilizou o percentual de sífilis congênita, mas identificou que 2 das crianças que moravam com as mães no ambiente prisional foram diagnosticadas com sífilis. Batista et al. (2020) e Gouvêa, Corrêa e Santos (2019) não avaliaram a transmissão vertical de sífilis e a incidência de sífilis congênita.

De um modo geral, o Quadro 3 evidencia três pontos: a alta prevalência de sífilis em gestantes privadas de liberdade, a alta taxa de transmissão vertical de sífilis nesse grupo e a escassez de pesquisas acerca do combate à sífilis congênita no ambiente prisional.

\section{CONCLUSÕES}

Por meio da revisão da literatura apresentada neste artigo, constata-se que as mulheres privadas de liberdade são uma população chave para o combate à sífilis congênita e à sífilis em gestantes. Isso porque a prevalência de sífilis em gestantes, a taxa de transmissão vertical de sífilis e a incidência de sífilis congênita nos estudos realizados nesse grupo apresentaram números bastante elevados, principalmente, quando comparados aos dados de mulheres livres.

Outro ponto importante discutido é que os casos de sífilis congênita e em gestantes, que permeiam esse grupo, poderiam ter sido evitados por meio de diagnóstico precoce e tratamento adequado durante o pré-natal. Portanto, a melhor estratégia para combater a sífilis congênita no ambiente prisional é garantir que todas as gestantes privadas de liberdade tenham acesso a um pré-natal adequado, incluindo todas as ações de combate à sífilis oferecidas às gestantes livres. Desse modo, é possível reduzir de forma consistente a prevalência de sífilis em gestantes, a taxa de transmissão vertical de sífilis e a incidência de sífilis congênita no ambiente prisional.

Nesse sentido, esse artigo corrobora com as ideias de Gouvêa, Corrêa e Santos (2019), indicando a intensificação da Política Nacional de Atenção Integral à Saúde das Pessoas Privadas de Liberdade no Sistema Prisional como um dos caminhos para combater a sífilis congênita e a sífilis em gestantes no sistema prisional brasileiro.

Por fim, esse artigo evidencia a necessidade de fortalecer as ações de vigilância epidemiológica da sífilis no sistema prisional brasileiro e de intensificar o cuidado integral, permitindo diagnóstico precoce e tratamento rápido e adequado para as pessoas privadas de liberdade. Além disso, constata-se uma demanda por educação em saúde para esse grupo, visto que falta de acesso às informações educativas acerca de doenças como a sífilis distanciam essas pessoas dos mecanismos de prevenção de sífilis adotados para a população em geral. 


\section{REFERÊNCIAS}

BATISTA, M. I. H. D. M. et al. Alta prevalência de sífilis em unidade prisional feminina do Nordeste brasileiro. Einstein,

São Paulo, v. 18, 2020.

BLANK, S. et al. New approaches to syphilis control. Finding opportunities for syphilis treatment and congenital syphilis prevention in a women's correctional setting. Sexually Transmitted Diseases. v. 24, n. 4, p. 218-226, 1997.

BRASIL. Ministério da Saúde. Secretaria de Vigilância em Saúde. Departamento de DST, Aids e Hepatites Virais. Boletim Epidemiológico - Sífilis 2019. Brasília: Ministério da Saúde, 2019. Disponível em http://www.aids.gov.br/pt-br/pub/2019/boletimepidemiologico-sifilis-2019. Acesso em: 20 jul.2020.

BRASIL. Ministério da Justiça. Departamento Penitenciário Nacional. Diretrizes para a Convivência Mãe-Filho/a no Sistema Prisional. Brasília: Ministério da Justiça, 2016.

BRASIL. Ministério da Saúde. Secretaria de Vigilância em Saúde. Programa Nacional de DST/AIDS. Diretrizes para controle da sífilis congênita: manual de bolso. Brasília: Ministério da Saúde, 2006.

BRASIL. Ministério da Justiça e Segurança Pública. Departamento Penitenciário Nacional. Levantamento Nacional de Informações Penitenciárias INFOPEN- Mulheres. Brasília: Ministério da Justiça, 2017.

BRASIL. Ministério da Saúde. Portaria Interministerial $\mathbf{n}^{\circ}$ 1, de 02 de janeiro de 2014. Institui a Política Nacional de Atenção à Saúde das Pessoas Privadas de Liberdade no Sistema Prisional no âmbito do Sistema Único de Saúde. Brasília: Ministério da Saúde, 2014.

\section{BRASIL. TED 54 - TERMO DE EXECUÇÃO DESCENTRALIZADA.}

Instrumento por meio do qual é ajustada a descentralização de crédito entre órgãos e/ou entidades integrantes dos Orçamentos Fiscal e da Seguridade Social da União, para execução de ações de interesse da unidade orçamentária. Brasilia: [s.n.], 2017.

CORREA, M. E. et al. High prevalence of Treponema pallidum infection in Brazilian prisoners. The american journal of tropical medicine and hygiene, v. 97, n. 4, p. 1078-1084, 2017.

DOMINGUES, R. M. S. M. et al. Prevalence of syphilis and HIV infection during pregnancy in incarcerated women and the incidence of congenital syphilis in births in prison in Brazil. Cad Saude Publica, v. 33, n. 11, p. e00183616, 2017. 
GÉTAZ, L. et al. Congenital syphilis in 2 children in a Bolivian prison.

Revista Española de Sanidad Penitenciaria, v. 19, n. 3, p. 98-100, 2017.

GEWANDSZNAJDER, F. O que é o método científico. São Paulo:

Pioneira, 1989.

GIL, A. C. Métodos e técnicas de pesquisa social. São Paulo:

Atlas, 1999.

GOUVÊA, A. N.; CORRÊA, R. S.; SANTOS, B. O. M. F. Incidência de sífilis e HIV em gestantes privadas de liberdade no Rio de Janeiro.

Brazilian Journal of Health Review, v. 2, n. 3, p. 1596-1603, 2019.

LAKATOS, E. M.; MARCONI, M. de A. Metodologia do trabalho científico. São Paulo: Atlas, 1991.

LEAL, M. D. C. et al. Nascer na prisão: gestação e parto atrás das

grades no Brasil. Ciência \& Saúde Coletiva, n. 21, p. 2061-2070, 2016.

MATTHIAS, J. M. et al. Effectiveness of prenatal screening and treatment to prevent congenital syphilis, Louisiana and Florida, 2013-2014. Sexually transmitted diseases, v. 44, n. 8, p. 498, 2017.

SOUZA, A. T. da S. Prevalência da sífilis e fatores de risco associados em internos do sistema prisional do Piauí. Dissertação (Mestrado) - Universidade Federal do Piauí, Programa de Pós-Graduação em Enfermagem, Teresina, 2017. 